\title{
Massive gastric polyposis associated with a germline SMAD4 gene mutation
}

\author{
Eline Soer ${ }^{1}$ (D) Wouter H. de Vos tot Nederveen Cappel $^{1} \cdot$ Marjolijn J. L. Ligtenberg $^{4} \cdot$

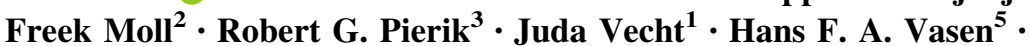 \\ Antoine Flierman ${ }^{1}$
}

Published online: 10 July 2015

(C) Springer Science+Business Media Dordrecht 2015

\begin{abstract}
Juvenile polyposis syndrome (JPS) is a rare autosomal dominant disorder characterized by the development of multiple hamartomatous polyps in the gastrointestinal tract. Polyps are most common in the colorectum (98\% of patients) and the stomach (14\%). Causative mutations for JPS have been identified in two genes to date, SMAD4 and BMPRIA. SMAD4 mutations are associated with a higher incidence of gastric polyposis. In this case report, we describe two patients with massive gastric polyposis associated with a SMAD4 mutation. Both presented with anaemia and both had colonic polyps. Initial endoscopic findings revealed giant rugal folds suggestive of Ménétrier disease. However, as other possible gastropathies could not be differentiated on the basis of histology, a definitive diagnosis of JPS required additional mutation analysis. In patients with polyposis predominant in or limited to the stomach, establishing a diagnosis based solely on the pathological features of polyps can be
\end{abstract}

Eline Soer

elinesoer@gmail.com

1 Department of Gastroenterology and Hepatology, Isala Clinics, P.O. Box 10400, 8000 GK Zwolle, The Netherlands

2 Department of Pathology, Isala Clinics, Zwolle, The Netherlands

3 Department of Surgery, Isala Clinics, Zwolle, The Netherlands

4 Department of Human Genetics, Radboud University Medical Center, Nijmegen, The Netherlands

5 The Netherlands Foundation for the Detection of Hereditary Tumors, Leiden, The Netherlands challenging due to difficulties in differentiating JPS from other hypertrophic gastropathies. Mutation analysis should be considered early in the diagnostic process in cases of suspected juvenile polyposis, thus facilitating rapid diagnosis and adequate follow-up.

Keywords Gastric polyposis - Juvenile polyposis · SMAD4 mutation

\section{Introduction}

Juvenile polyposis syndrome (JPS) is a rare autosomal dominant disorder with a prevalence of approximately one per 100,000 . JPS shows variable penetrance and is characterized by the development of multiple hamartomatous polyps in the gastrointestinal tract, which develop into malignant lesions in approximately $20 \%$ of cases. Most patients develop polyps in the colorectum (98\%), followed by locations including the stomach (14\%), small intestine $(7 \%)$ and duodenum $(2 \%)[1,2]$.

Approximately $75 \%$ of JP patients have a family history of JPS, with the remaining $25 \%$ thought to have de novo mutations [3]. Two causative genes, SMAD4 and BMPRIA, have been identified to date, with germline mutations in each gene explaining around $20 \%$ of the prevalence. The remaining $60 \%$ of patients suspect for JPS have no known mutation. Patients with a SMAD4 mutation show a higher prevalence of gastric polyposis compared to patients with a BMPR1A mutation [4-6], and most SMAD4 patients also have hereditary haemorrhagic telangiectasia [7, 8]. Establishing a diagnosis in patients with juvenile polyposis predominant in or limited to the stomach can be challenging due to difficulties in differentiating JPS from other hypertrophic gastropathies. 


\section{Case report 1}

A 54-year-old female was referred to our clinic for a second opinion after numerous polyps of the corpus and cardia were found during an upper oesophagogastroduodenoscopy (EGD) in 2001, which was performed due to persistent iron deficiency anaemia ( $\mathrm{Hb}$ 6.9). The patient previously underwent an EGD (1993) following a stomach ache, which revealed multiple polyps in the antrum and corpus region of the stomach. Biopsies of the polyps revealed chronic inflammation with eosinophilia, but no further action was undertaken at the time. Although the patient had no complaints during the first outpatient visits in 2001, anaemia recurred as soon as iron supplementation was stopped. She had no hypoalbuminemia, nor did she suffer from oedema, which are symptoms often accompanying Ménétrier disease (MD). There were no phenotypic hallmarks of Cronkhite-Canada syndrome, such as nail abnormalities or alopecia. Her family history revealed a son who had undergone laparoscopic bowel surgery at age 8 for duodenal polyps and at age 29 for rectal polyps. Her daughter had no relevant medical history.

The patient had 15 polyps removed during colonoscopic examination and histological examination showed adenomas with low grade dysplasia. The EGD was repeated and revealed numerous polyps and giant rugal folds, especially in the gastric body, from which biopsies were taken. Histological evaluation showed foveolar hyperplasia affecting the gastric body together with glandular atrophy. Helicobacter pylori status was negative. While the endoscopic and histological findings were suggestive of MD, other findings argued against it. The lack of hypoalbumenia and oedema, as well as the presence of numerous colon polyps, suggested other hypertrophic gastropathies could be the cause.

The patient underwent yearly colonoscopies and EGD's. Unfortunately, during this time no special attention was given to the possible presence of small intestine polyps or micro vessel telangiectasia. These findings could have helped bring a JPS to light earlier.

In 2004, the patient underwent a right-sided hemicolectomy for multiple adenomas in the ascending part of the colon and caecum which could not be completely removed endoscopically. Histology showed multiple tubulovillous adenomas with low grade dysplasia.

A complete gastrectomy with oesophagojejunostomy was carried out in 2006 as a consequence of recurrent anaemia $(\mathrm{Hb}<6)$, hypoalbuminemia (serum albumin $<30$ ) and suspicion of malignancy. Macroscopic examination of the resected stomach revealed numerous polyps with a coral-like appearance (Fig. 1), with evidence of extensive polyp hyperplasia on histological examination and a maximal polyp diameter of $5 \mathrm{~cm}$. No dysplasia was found and

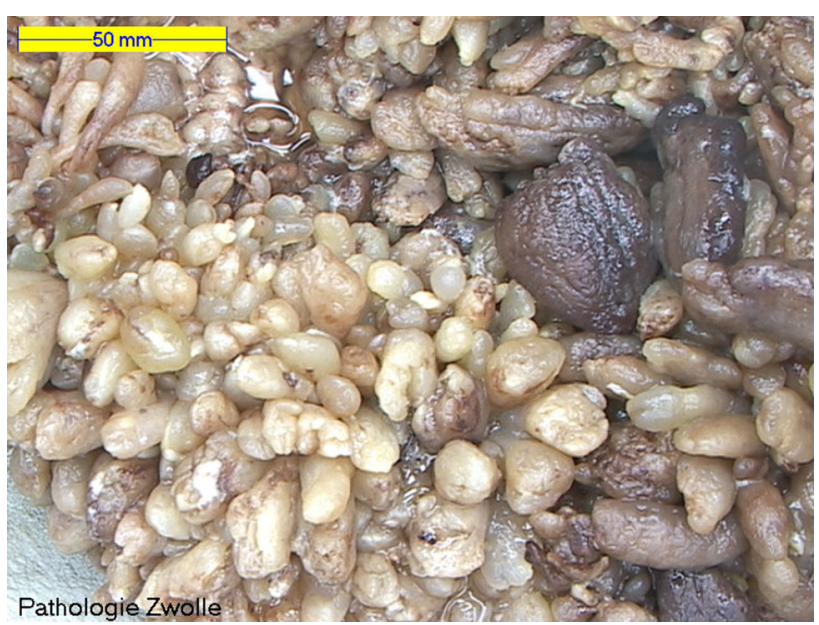

Fig. 1 Resected stomach of the first patient. Macroscopic examination shows numerous polyps with a coral-like appearance

the disorderly architecture did not suggest Ménétriers disease. The patient was then referred to the department of Clinical Genetics, where tests for germline BMPRIA, SMAD4 and PTEN mutations were carried out. No mutation was found at the time.

In 2007 , the patient was admitted with a pathological fracture of the left femur. Histology revealed a metastatic adenocarcinoma with an unknown primary location. CT showed metastases to the liver and bones and additional immunohistochemical staining was suggestive of a gastroenterological primary tumor. The patient died shortly after, before a conclusive diagnosis could be established. Her family did not approve an autopsy to establish the primary site of carcinoma.

In 2008, the patient's son was referred to our clinic for surveillance on the basis of his and his mother's medical history. EGD showed a normal stomach but a pedunculated polyp in the duodenal bulbus which was subsequently removed and shown to be a hyperplastic polyp on histological examination. During colonoscopic surveillance two juvenile polyps were removed from the caecal and sigmoid part of the colon. In 2010, a small adenoma in the hepatic flexure was removed. Capsule endoscopy of the small bowel showed no other abnormalities.

Genetic analysis carried out in 2010 identified a complete deletion of the SMAD4 gene. A revision of the histology was performed and in this new context gastric juvenile polyposis was diagnosed. An echo sonography and CT of the abdomen revealed no vascular malformations. Contrast echocardiography revealed a grade 3 pulmonary left-to-right shunt.

As original mutation testing in 2004 did not yet include analysis of exon deletions and duplications, germline DNA from the patient's mother was retested and the same SMAD4 gene deletion identified. 


\section{Case report 2}

In 2012, a 48-year-old male was evaluated for symptomatic iron deficiency anaemia ( $\mathrm{Hb}$ 3.7) in another hospital. Gastric polyposis and enlarged rugal folds were seen on EGD. The patient had no relevant medical history and no family history of gastrointestinal disease. MD was considered the most likely diagnosis.

The patient had no gastrointestinal complaints on referral to our clinic. With the exception of iron deficiency, laboratory testing showed no abnormalities and $H$. pylori infection was ruled out by serological testing. There was no hypoalbuminemia or oedema, nor were there phenotypic hallmarks associated with Cronkhite-Canada syndrome. EGD and linear endoscopic ultrasound (EUS) showed a stomach with enlarged rugal folds and a large tumor-like abnormality in the large curvature, with erratic projections. The mucosa and submucosa were greatly thickened, with an intact muscularis propria.

Histological examination of biopsies from the antrum, fundus and cardia showed marked cystic dilatation of the foveolae, glandular atrophy, inflammation, multiple telangiectasia and oedema of the lamina propria. There was no dysplasia, intraepithelial lymphocytic infiltration, antral sparing or smooth muscle hypertrophy. CT of the abdomen showed no growth of the lesion beyond the lamina propria and no metastases. During colonoscopic examination two polyps were removed and histology revealed a tubulovillous and inflammatory polyp. Based on these findings juvenile polyposis was considered most likely, but Cowden syndrome and hyperplastic gastropathies such as MD could not be excluded.

SMAD4 gene analysis revealed a deletion of exons $1-8$, confirming JPS. CT showed no signs of cerebral, abdominal or pulmonary telangiectasia. Oral and parenteral iron supplements were started, with moderate success (Hb levels 5.5-7.7). Capsule endoscopy showed no abnormalities in the small bowel. Annual surveillance by EGD has been implemented, including stomach debulking, with the benign lesion showing evidence of growth over the last 2 years. As the patient is currently free of complaints and the malignant potential of the lesion is largely unknown, a possible indication for gastrectomy is still under discussion.

\section{Discussion}

\section{Summary of patients}

We described two patients with massive gastric polyposis associated with deletions of the SMAD4 gene. Both presented with anaemia and both had colonic polyps. Juvenile polyposis predominant in or limited to the stomach (GJP) is a rare condition and is linked to $S M A D 4$ mutations. It is debatable whether GJP is a subtype of JP or a separate disease entity. A diagnosis of GJP should be considered whenever a mixed, hypertrophic and polypoid gastropathy is encountered [9]. However, accurate diagnosis in patients with gastric foveolar hyperplasia can be difficult because polyps show wide histological variation. Careful correlation of clinical, laboratory, imaging and pathological findings is required before a diagnosis can be reached, and even then findings can show broad overlap between the various hyperplastic gastropathies. These difficulties are illustrated by our first patient, for whom a correct diagnosis required several years. In both cases the identification of a SMAD4 mutation was required to achieve a definitive diagnosis.

\section{Juvenile polyposis syndrome}

JPS is a rare autosomal dominant disorder with a prevalence of approximately one in 100,000 . The WHO criteria for diagnosis of JPS require one of the following:

1. More than five juvenile polyps in the colon or rectum; or

2. Juvenile polyps throughout the gastrointestinal tract; or

3. Any number of juvenile polyps in a person with a family history of juvenile polyposis.

The syndrome may also be confirmed by the identification of a genetic defect associated with JPS.

The two genes known to be involved in juvenile polyposis, SMAD4 and BMPRIA, both encode proteins with roles in TGF- $\beta$ signal transduction pathways $[10,11]$. Germline mutations in genes associated with the signalling pathways of the transforming growth factor- $\beta$ (TGF- $\beta$ ) super family can lead to a range of heritable disorders such as JPS, but also including the vascular disorders hereditary haemorrhagic telangiectasia (HHT) and primary pulmonary hypertension. In the case of combined JPS/HHT syndrome, SMAD4 pathogenic variants are primarily located within the MH2 domain (exons 8-11), although some pathogenic variants in other exons have been observed.

Telangiectasia in HHT occurs in the skin and in the mucosal lining of the nose and gastrointestinal tract, giving rise to nosebleeds and acute and chronic digestive tract bleeding. Arteriovenous malformations (AVM) may occur in larger organs such as the lungs, liver and the brain. Common complications due to AVM include stroke (ischemic and haemorrhagic) and brain abscesses. Treatment focuses on reducing bleeding from blood vessel lesions, and sometimes surgery or other targeted interventions to remove arteriovenous malformation in organs. 
Approximately one in three patients has chronic anaemia and chronic bleeding often requires iron supplements.

A recent study reported a high prevalence of massive gastric polyposis among SMAD4 mutation carriers. Friedl et al. described massive gastric polyposis in seven patients with SMAD4 mutations, four of whom required partial or total gastrectomy. Furthermore, two patients had several relatives who also suffered from massive gastric polyposis and who had undergone partial or total gastrectomy due to polyposis. In contrast, severe forms of gastric polyposis were not reported in JPS families with BMPR $1 A$ mutations or in families without identified mutations. Age-related penetrance was also suggested in the report [12].

The diagnosis of gastric juvenile polyps exclusively on the basis of histology is difficult due to the unusual pattern of polyps which can mimic hyperplastic polyps. Typical juvenile polyps show a normal epithelium with a dense stroma, an inflammatory infiltrate, and a smooth surface with dilated, mucus-filled cystic glands in the lamina propria. Muscle fibers and the proliferative characteristics of adenomas are typically absent in juvenile polyps.

Initially, both our patients were suspected to have MD, although different gastropathies were also considered. The foveolar hyperplasia and glandular atrophy seen on histology is found in both MD and JPS. The unravelling of these diagnoses was recently described by Rich et al. [13] in a series of 48 patients suspect for MD. The disease was confirmed in only 25 of the patients and the most common mimics of $\mathrm{MD}$ in the remaining patients were various forms of polyps and polyposis syndromes (13/23), with JPS being the most common (3/23). In this particular series non-MD cases tended to show more severe anaemia, whereas genuine MD cases had a lower median serum albumin.

\section{Clinical implications}

In light of the difficulty involved in distinguishing between the various types of hyperplastic gastropathies, the possibility of implementing genetic testing for SMAD4, BMPRIA and PTEN mutations in an earlier phase of the diagnostic process is worth considering. Identification of the underlying gene defect has important implications for screening methods and the management of polyps.

\section{Managing JPS}

Risk of GI cancers in families with JPS ranges from 9 to $50 \%$, the risk of colorectal cancer is reportedly between 10 and $38 \%$, and the risk of gastric cancer is around $21 \%$, although no formal risk analysis for gastric cancer has yet been undertaken [14]. However, it is logical to assume that the risk of gastric cancer in SMAD4 mutation carriers is higher than the $21 \%$ reported for the JPS population overall.

Surveillance of the colorectum is recommended at intervals of 1-2 years from age 15, or earlier in cases where a patient develops symptoms. Surveillance should probably continue up to the age of 70 [15]. As many polyps are located in the right colon, the complete colon should be visualized during endoscopy, and to reduce the risk of bleeding, intestinal obstruction and colon cancer, any polyps should be removed. Prophylactic surgery should also be considered in cases with multiple polyps that cannot be controlled endoscopically.

Effective surveillance of the upper GI tract is less well understood. Capsule endoscopy to evaluate the small bowel should probably be carried out at least once. Upper gastrointestinal surveillance by endoscopy is recommended at intervals of 1-2 years from age 25 onwards [15, 16]. However, there are no data to support this approach and uncertainty as to potential benefits should be made clear to the patient.

Whether (partial) gastrectomy can be advised in patients with severe gastric polyposis is a difficult question. The removal of (part of) the stomach seems justifiable when polyposis gives rise to symptoms refractory to therapy, such as iron deficiency anaemia or hypoproteinemia. However, in cases such as that of our second patient, who is currently asymptomatic, it is difficult to be certain that yearly EGD is sufficient to manage the risk of gastric cancer. Endoscopic treatment of gastric polyps in the case of juvenile polyposis, or any other case of massive gastric polyposis, has never been described.

In patients with a germline SMAD4 mutation, screening for signs of hereditary haemorrhagic telangiectasia should be considered and should include chest radiography for arteriovenous malformations, magnetic resonance imaging of the brain, and liver sonography.

\section{Conclusion}

We described two patients with massive gastric polyposis. Establishing a definitive diagnosis based solely on the pathological features of the polyps is often not possible in these cases, due to the wide variety of histologies displayed by polyps. Because mutation of the SMAD4 and BMPRIA genes appears to result in different and distinct phenotypes, accurate identification of the underlying genetic defect is important. Therefore, as soon as a suspicion of juvenile polyposis is raised, mutation analysis of SMAD4 and BMPRIA should be considered as an aid to quick and accurate diagnosis. 


\section{References}

1. Desai DC, Neal KF, Talbot IC, Nodgson SV, Philips RKS (1995) Juvenile polyposis. Br J Surg 82:14-17

2. Scott-Conner CEH, Hausmann M, Hall TJ, Skelton DS, Anglin BL, Subramony C (1995) Familial juvenile polyposis: patterns of recurrence and implications for surgical management. J Am Coll Surg 181:407-413

3. Larsen Haidle J, Howe JR (1993-2014) Juvenile polyposis syndrome. 2003 May 13 [Updated 2014 May 22]. In: Pagon RA, Adam MP, Ardinger $\mathrm{HH}$ et al (eds) GeneReviews ${ }^{\circledR}$ [Internet]. University of Washington, Seattle

4. Howe JR, Sayed MG, Ahmed AF, Ringold J, Larsen-Haidle J, Merg A et al (2004) The prevalence of MADH4 and BMPR1A mutations in juvenile polyposis and absence of BMPR2, BMPR1B, and ACVR1 mutations. J Med Genet 41:484-491

5. Shikata K, Kukita Y, Matsumoto T, Esaki M, Yao T, Mochizuki Y, Hayashi K, Iida M (2005) Gastric juvenile polyposis associated with germline SMAD4 mutation. Am J Med Genet 134A:326-329

6. Handra-Luca A, Condroyer C, de Moncuit C, Tepper M, Fléjou JF, Thomas G, Olschwang S (2005) Vessels' morphology in SMAD4 and BMPR1A-related juvenile polyposis. Am J Med Genet 138A:113-117

7. O’Malley M, LaGuardia L, Kalady MF, Parambil J, Heald B, Eng C, Church J, Burke CA (2012) The prevalence of hereditary hemorrhagic telangiectasia in juvenile polyposis syndrome. Dis Colon Rectum 55:886-892

8. Schwenter F, Faughnan ME, Gradinger AB, Berk T, Gryfe R, Pollett A, Cohen Z, Gallinger S, Durno C (2012) Juvenile polyposis, hereditary hemorrhagic telangiectasia, and early onset colorectal cancer in patients with SMAD4 mutation. J Gastroenterol 47:795-804
9. Pintiliciuc OG, Heresbach D, de-Lajarte-Thirouard AS, Dugast C, Reignier A, Cottereau J, Bretagne JF (2008) Gastric involvement in juvenile polyposis associated with germline SMAD4 mutations: an entity characterized by a mixed hypertrophic and polypoid gastropathy. Gastroen Clin Biol 32(5):445-450

10. Haramis AP, Begthel H, van den Born M, van Es J, Jonkheer S, Offerhaus GJ et al (2004) De novo crypt formation and juvenile polyposis on BMP inhibition in mouse intestine. Science 303(5664):1684-1686

11. Gallione C, Aylsworth AS, Beis J, Berk T, Bernhardt B, Clark $\mathrm{RD}$ et al (2010) Overlapping spectra of SMAD4 mutations in juvenile polyposis (JP) and JP-HHT syndrome. Am J Med Genet 152A:333-339

12. Friedl W, Uhlhaas S, Schulmann K, Stolte M, Loff S, Back W, Mangold E et al (2002) Juvenile polyposis: massive gastric polyposis is more common in MADH4 mutation carriers than in BMPR1A mutation carriers. Hum Genet 111:108-111

13. Rich A, Zuluaga Toro T, Tanksley J, Fishe WH, Lind CD, Ayers GD (2010) Distinguishing Ménétrier's disease from its mimics. Gut 59(12):1617-1624

14. Howe JR, Mitros FA, Summers RW (1998) The risk of gastrointestinal carcinoma in familial juvenile polyposis. Ann Surg Oncol 5:751-756

15. Dunlop MG (2002) Guidance on gastrointestinal surveillance for hereditary non-polyposis colorectal cancer, familial adenomatous polyposis, juvenile polyposis, and Peutz-Jeghers syndrome. Gut 51(Suppl 5):V21-V27

16. Postgate AJ, Will OC, Fraser CH, Fitzpatrick A, Philips RK, Clark SK et al (2009) Capsule endoscopy for the small bowel in juvenile polyposis syndrome: a case series. Endoscopy 41(11):1001-1004 